\title{
Effects of upper airway carbon dioxide on upper airway resistance and muscle activity in young guinea-pigs
}

\author{
A.K. Curran, K.D. O'Halloran, A. Bradford
}

\begin{abstract}
Effects of upper airway carbon dioxide on upper airway resistance and muscle activity in young guinea-pigs. A.K. Curran, K.D. O'Halloran, A. Bradford. (C)ERS Journals Ltd 2000. ABSTRACT: The upper airway (UA) of adult animals is known to contain carbon dioxide-sensitive receptors and $\mathrm{UA} \mathrm{CO}_{2}$ reflexly affects breathing, $\mathrm{UA}$ dilator muscle activity and $U A$ resistance. These effects may function in the control of $U A$ patency. There is evidence that some UA reflexes are stronger in young than in adult animals, but it is not known whether $\mathrm{CO}_{2}$-sensitive receptors are present in the $\mathrm{UA}$ of young animals, and the effects of $\mathrm{UA} \mathrm{CO}_{2}$ on $\mathrm{UA}$ resistance and on $\mathrm{UA}$ dilator muscle activity have not been investigated in young animals.

The responses of ventilation, $U A$ resistance and geniohyoid muscle electromyographic activity to warm air containing $10 \% \mathrm{CO}_{2}$ applied to the isolated $\mathrm{UA}$ were measured in anaesthetized, vagotomized young guinea-pigs breathing spontaneously through a low-cervical tracheostomy.

Upper airway carbon dioxide caused an increase in ventilation $(46.7 \pm 16.3$ to $49.9 \pm 16.8 \mathrm{~mL} \cdot \mathrm{min}^{-1} \cdot 100 \mathrm{~g}$ body weight $\left.{ }^{-1}\right)$ and upper airway resistance $(56.8 \pm 14.8$ to $\left.63.7 \pm 17.7 \mathrm{cmH}_{2} \mathrm{O} \cdot \mathrm{L}^{-1} \cdot \mathrm{s}^{-1} \cdot \mathrm{kg} \mathrm{body} \mathrm{weight}^{-1}\right)$. Similar effects were obtained following vagotomy. Geniohyoid activity became apparent following vagotomy and this activity was reduced by upper airway carbon dioxide. These responses were abolished by topical anaesthesia of the upper airway. This suggests that the reflexes seen are due to carbon dioxide-sensitive receptors in the upper airway.

Eur Respir J 2000; 15: 902-905.
\end{abstract}

A variety of carbon dioxide-sensitive receptors have been identified in the upper airway (UA) of adult cats [1] and $\operatorname{dogs}$ [2], and introducing $\mathrm{CO}_{2}$ into the isolated $\mathrm{UA}$ in adult animals causes a number of reflex effects such as a reduction in ventilation $[3,4]$, an increase in UA muscle activity $[3,4]$ and an increase in laryngeal resistance [5]. These effects have been suggested to play a role in the regulation of UA patency $[3,5]$.

It is not known whether the UA contains $\mathrm{CO}_{2}$-sensitive receptors in young animals, although it has been shown previously that passing $\mathrm{CO}_{2}$ into the isolated UA causes tachypnoea in young guinea-pigs [6]. There is evidence that some UA reflexes are stronger in young animals compared to adults [7-9], suggesting that such reflexes may play a more important role in regulating UA patency in the young. The importance of studying such reflexes in the young is also relevant to their greater vulnerability to UA collapse and to obstructive sleep apnoea in infants and possibly sudden infant death [10]. However, the effects of UA $\mathrm{CO}_{2}$ on UA resistance or on UA muscle activity have not been investigated in young animals.

Therefore, the present study was undertaken in order to test the hypothesis that applying $\mathrm{CO}_{2}$ to the isolated UA affects UA resistance and UA dilator muscle activity in young guinea-pigs. Guinea-pigs are more mature when young compared to many species [11]. However, UA reflexes are very different in 10-14-day-old compared to adult guinea-pigs [12] and there are also maturational differences in local airway responses between 15-day-old and adult guineapigs [13].
Dept of Physiology, Royal College of Surgeons in Ireland, St. Stephen's Green, Dublin 2, Ireland.

Correspondence: A. Bradford, Dept of Physiology Royal College of Surgeons in Ireland, St Stephen's Green, Dublin 2, Ireland. Fax: 35314022447

Keywords: Airway carbon dioxide airway resistance

geniohyoid

Received: November 301998

Accepted after revision June 151999

This work was supported by grants from the Health Research Board (Ireland), the Royal College of Surgeons in Ireland and the Wellcome Trust.

\section{Materials and methods}

The methods used for UA isolation and application of UA airflow have been described previously $[6,14]$. Briefly, 15 young guinea-pigs (aged 10-16 days; body mass 150$250 \mathrm{~g})$ were anaesthetized with urethane $(2 \mathrm{~g} \cdot \mathrm{kg}$ body weight ${ }^{-1}$ i.p.) and placed supine on a thermostatically controlled heating blanket to maintain body temperature at $37^{\circ} \mathrm{C}$. A cannula was inserted into a low-cervical tracheostomy through which the animal breathed spontaneously. Tracheal airflow was continuously recorded using a heated pneumotachograph (Hans Rudolph, KS, USA) and a differential pressure transducer (Validyne DP 15, CA, USA) attached to this cannula, and the signal was integrated to give tidal volume. A common carotid artery was cannulated and connected to a pressure transducer (Validyne DP 215) to measure systemic arterial blood pressure. This procedure interferes with the blood flow to the ipsilateral carotid body and sinus. However, blood pressure remained stable throughout the experiments. A jugular vein was cannulated to administer supplemental doses of anaesthetic as required.

A cannula was inserted into a high-cervical tracheostomy, and was pushed cranially to lie just caudal to the level of the cricoid cartilage. A high-pressure source produced a steady flow of $5-10 \mathrm{~mL} \cdot \mathrm{s}^{-1}$ of warmed humidified air or warmed humidified air containing $10 \% \mathrm{CO}_{2}$ which was delivered to the UA in an expiratory direction and passed over the larynx and pharynx to exit through the nose and mouth. Although the UA normally receives airflow of 
alternating inspiratory and expiratory flow, the technique of continuous expiratory flow has been used extensively to study UA receptor and reflex function [2, 4-7, 9, 14-17]. In order to maximize any responses obtained $10 \% \mathrm{CO}_{2}$ was used since the receptor and reflex responses to UA $\mathrm{CO}_{2}$ have been shown to be greater with $10 \% \mathrm{CO}_{2}$ than with the more physiological level of $5 \% \mathrm{CO}_{2}[4,16,17]$. UA airflow was recorded using a pneumotachograph (Hans Rudolph) in series with the high-cervical tracheostomy cannula and connected to a pressure transducer (Validyne DP 15). The temperature of the UA airflow was monitored using a thermocouple microprobe (Physitemp, Model TH8 with probe IT21, time constant $0.08 \mathrm{~s}$, Clifton, USA) extended into the lumen of the UA cannula through a sidearm. Subglottic UA pressure was measured using a pressure transducer (Validyne DP 15) connected to a sidearm of the UA cannula and ranged $1-3 \mathrm{cmH}_{2} \mathrm{O}$. Mean values of UA pressure and flow were used to calculate UA resistance.

The vagus nerves were carefully exposed bilaterally, marked with short lengths of cotton thread and maintained intact for later section. Great care was taken during these procedures to avoid damage to the superior and recurrent laryngeal nerves. Fine bipolar copper wire electrodes were inserted into the geniohyoid muscle, a UA dilator, in order to record its electrical activity. The geniohyoid electromyograph wires were connected to an amplifier and integrator system (Neurolog system NL 900A, Digitimer, Welwyn Garden City, UK). The signal was band-pass filtered, rectified and amplified (Neurolog NL 104) before being integrated using a leaky integrator with a time constant of 50 ms (Neurolog NL 703). All signals were digitized and recorded using a commercial data acquisition system and stored for later analysis on a microcomputer.

\section{Protocol}

In all 15 animals, with the vagus nerves intact, variables were recorded continuously during application of warmed humidified air. Trials were performed by switching to warmed humidified air containing $10 \% \mathrm{CO}_{2}$ which was applied for $30-60 \mathrm{~s}$ before switching back to air alone. The vagus nerves were cut at the mid-cervical level and the $10 \%$ $\mathrm{CO}_{2}$ trial repeated. Following this, in nine of the 15 animals, a solution of $2 \%$ Xylocaine was applied to the entire $\mathrm{UA}$ for $10 \mathrm{~min}$, and the $10 \% \mathrm{CO}_{2}$ trial repeated.

\section{Data analysis}

The values for respiratory variables, UA resistance and geniohyoid muscle electromyographic activity were averaged from ten control breaths prior to the application of $10 \% \mathrm{CO}_{2}$ to the UA, from ten breaths during the trial period when the effect of the $10 \% \mathrm{CO}_{2}$ was greatest and from ten breaths following the removal of the $\mathrm{CO}_{2}$. Since there was a slight fall in UA resistance during inspiration and an increase during expiration, UA resistance was calculated as the mean of inspiratory plus expiratory resistance. Phasic geniohyoid integrated electromyographic activity was quantified as the height of the peak integrated signal from end-expiratory level in arbitrary units. The breath-tobreath variability of geniohyoid muscle activity was also quantified as the coefficient of variation, i.e. SD/mean ex- pressed as a percentage. This was carried out because geniohyoid electromyographic activity has been demonstrated to show marked breath-to-breath variability in adult rats [15] and cats [18]. Data are expressed as absolute values or as mean \pm SD percentage change with respect to control. The data was analysed for statistical significance using analysis of variance and Fisher's least significant difference test, with a $\mathrm{p}$-value of $<0.05$ being taken as significant.

\section{Results}

With the vagus nerves intact, there was no phasic electromyographic activity in the geniohyoid muscle. Switching the flow through the UA from air to air containing $10 \% \mathrm{CO}_{2}$ had no effect on UA airflow or temperature or on arterial blood pressure. The effects of $\mathrm{UA} \mathrm{CO}_{2}$ on ventilation and UA resistance are shown in table 1 . UA $\mathrm{CO}_{2}$ caused a significant increase in respiratory frequency (due to shortening of the expiratory time), minute ventilation and UA resistance.

Following section of the vagus nerves, phasic inspiratory geniohyoid electromyographic activity became apparent. The effects of $\mathrm{UA} \mathrm{CO}_{2}$ following vagotomy were similar to those before vagotomy, i.e. there was an increase in respiratory frequency, minute ventilation and UA resistance (table 1, fig. 1). In addition, $\mathrm{UA} \mathrm{CO}_{2}$ caused a decrease in peak integrated inspiratory geniohyoid electromyographic activity (fig. 1). This reduction in the activity of the geniohyoid, a UA dilator muscle, may have been associated with the increase in UA resistance. However, it was not possible to calculate the correlation of geniohyoid activity and UA resistance because the variances of the two sets of data were unequal. The increase in respiratory frequency, minute ventilation and UA resistance and the decrease in peak integrated inspiratory geniohyoid electromyographic activity were abolished by topical anaesthesia of the UA. The coefficient of variation of peak integrated geniohyoid electromyographic activity was unaffected by UA $\mathrm{CO}_{2}$ or by UA anaesthesia. Thus the coefficient was $20.4 \pm 7.4 \%$ in air and $20.5 \pm 7.1 \%$ with $10 \% \mathrm{CO}_{2}$. Following UA anaesthesia, the coefficient was $21.6 \pm 3.9 \%$ in air and $22.7 \pm 9.2 \%$ with $10 \% \mathrm{CO}_{2}$.

\section{Discussion}

The present results show that with intact vagus nerves applying $10 \% \mathrm{CO}_{2}$ to the lumen of the UA causes an increase in respiratory frequency, minute ventilation and UA resistance. Following vagotomy, similar effects were obtained and phasic inspiratory geniohyoid muscle electromyographic activity became apparent. This activity was inhibited by $\mathrm{UA} \mathrm{CO}_{2}$. The effects on ventilation confirm previous findings using the same preparation [14]. The absence of phasic geniohyoid muscle activity when the vagi were intact and its appearance when the vagi were cut also confirms previous observations [14], and is consistent with the fact that UA muscle activity is increased by vagotomy in adult animals $[19,20]$. It is also consistent with the possibility that the Hering-Breuer inflation reflex has a greater inhibitory effect on UA muscle activity in young animals compared to adult 
Table 1. - Effect of upper airway carbon dioxide on ventilation and upper airway resistance

\begin{tabular}{lcccccc}
\hline & $f \mathrm{R}$ breaths $\cdot \mathrm{min}^{-1}$ & $V \mathrm{~T} \mathrm{~mL} \cdot 100 \mathrm{~g}^{-1}$ & $V^{\prime} \mathrm{E} \mathrm{mL} \cdot \mathrm{min}^{-1} \cdot 100 \mathrm{~g}^{-1}$ & $t \mathrm{I} \mathrm{s}$ & $t \mathrm{E} \mathrm{s}$ & $R \mathrm{UA} \mathrm{cmH} \mathrm{cm}_{2} \mathrm{O} \cdot \mathrm{L}^{-1} \cdot \mathrm{s}^{-1} \cdot \mathrm{kg}^{-1}$ \\
\hline Vagus nerves intact & & & & & & \\
$\quad$ Control & $35.1 \pm 21.1$ & $1.1 \pm 0.4$ & $46.7 \pm 16.3$ & $0.7 \pm 0.4$ & $1.9 \pm 1.4$ & $56.8 \pm 14.8$ \\
$10 \% \mathrm{CO}_{2}$ & $39.6 \pm 22.0^{*}$ & $1.1 \pm 0.4$ & $49.9 \pm 16.8^{*}$ & $0.6 \pm 0.4$ & $1.5 \pm 1.2^{*}$ & $63.7 \pm 17.7^{*}$ \\
Vagi cut & & & & & & \\
$\quad$ Control & $21.3 \pm 10.1$ & $1.6 \pm 0.7$ & $40.8 \pm 14.6$ & $1.0 \pm 0.5$ & $2.4 \pm 1.2$ & $84.8 \pm 42.0$ \\
$10 \% \mathrm{CO}_{2}$ & $24.0 \pm 11.7^{*}$ & $1.5 \pm 0.6$ & $43.8 \pm 15.4^{*}$ & $1.0 \pm 0.5$ & $2.1 \pm 0.9^{*}$ & $93.6 \pm 42.2^{*}$ \\
After Xylocaine & & & & & \\
$\quad$ Control & $36.6 \pm 21.0$ & $1.2 \pm 0.5$ & $39.5 \pm 15.9$ & $0.6 \pm 0.3$ & $1.6 \pm 0.9$ & $116.2 \pm 28.1$ \\
$10 \% \mathrm{CO}_{2}$ & $36.3 \pm 20.7$ & $1.2 \pm 0.4$ & $39.3 \pm 15.8$ & $0.5 \pm 0.2$ & $1.6 \pm 0.9$ & $117.5 \pm 25.9$ \\
\hline
\end{tabular}

Data are presented as mean \pm SD. All parameters were measured before (contol) and during $\left(10 \% \mathrm{CO}_{2}\right)$ the application of $10 \% \mathrm{CO}_{2}$ to the isolated upper airway. $f \mathrm{R}$ : respiratory frequency; $V \mathrm{~T}$ : tidal volume; $V^{\prime} \mathrm{E}$ : minute ventilation; $t \mathrm{I}$ : inspiratory time; $t \mathrm{E}$ : expiratory time; $R \mathrm{UA}$ : upper airway resistance. ${ }^{*}$ : $\mathrm{p}<0.05$ versus control.

animals since geniohyoid activity was completely absent in the presence of intact vagi in the present experiments.

Cutting the vagi had no effect on the ventilatory and UA resistance responses to $\mathrm{UA} \mathrm{CO}_{2}$. Since vagotomy abolishes recurrent laryngeal afferent activity, this suggests that the responses are not due to afferents in the recurrent laryngeal nerves. The lack of effect of vagotomy on these responses may also suggest that Hering-Breuer inflation receptors have little effect on these reflexes. However, this interpretation must be viewed cautiously since vagotomy affected baseline air values and this may have influenced the subsequent responses to $\mathrm{UA} \mathrm{CO}_{2}$ following section of the vagi.

The effects of $\mathrm{UA} \mathrm{CO}_{2}$ on UA resistance or muscle activity have not been investigated previously in young animals. UA $\mathrm{CO}_{2}$ caused an increase in UA resistance and a decrease in geniohyoid muscle activity. The coefficient of variation of geniohyoid muscle activity is a measure of its breath-to-breath variability $[15,18]$ and the values obtained in the present experiments are substantially greater than those reported for UA muscle activity in adult cats [18] and rats [15]. This greater variability of UA muscle activity in young guinea-pigs compared to adult animals may contribute to the greater vulnerability of the young to UA collapse [10]. Since $\mathrm{UA} \mathrm{CO}_{2}$ reduced geniohyoid muscle

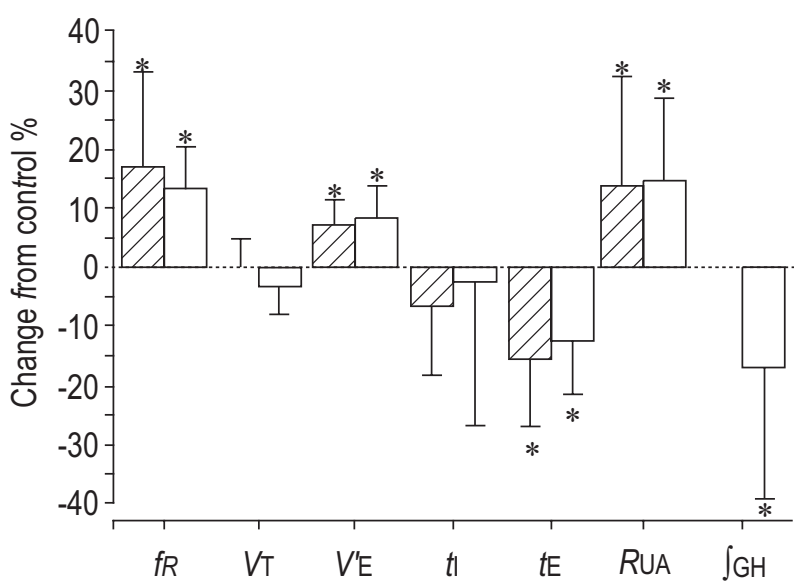

Fig. 1. - Effects of upper airway carbon dioxide on resiratory frequency $(f \mathrm{R})$, tidal volume $(V \mathrm{~T})$, minute ventilation $\left(V^{\prime} \mathrm{E}\right)$, inspiratory time $(t \mathrm{I})$, expiratory time $(t \mathrm{E})$, upper airway resistance $(R \mathrm{UA})$ and peak integrated geniohyoid electromyographic activity $(\mathrm{GH})$. Data are presented as mean \pm SD change from the value obtained in air (control) before $(\mathbb{Z})$ and after $(\square)$ vagotomy. $*$ : $\mathrm{p}<0.05$ versus control. activity, an effect on the variability of this activity might also have been anticipated. However, UA $\mathrm{CO}_{2}$ had no effect on the coefficient of variation of geniohyoid muscle activity. Since the geniohyoid muscle is a UA dilator [21], the increase in UA resistance may have been caused by the decrease in geniohyoid activity. A similar effect of $\mathrm{UACO}_{2}$ on UA resistance in adult rats in which laryngeal but not supraglottic resistance was increased has been described previously [5]. Furthermore, geniohyoid muscle activity is unaffected by UA $\mathrm{CO}_{2}$ in adult rats [15]. Therefore, although the inhibition of geniohyoid activity may have contributed to the increase in UA resistance in the present experiments, other sites of resistance may have been involved since the overall resistance of the sub- and supraglottic airway was measured and since UA resistance was increased by $\mathrm{UA} \mathrm{CO}_{2}$ in the absence of geniohyoid activity.

The inhibitory effect of $\mathrm{UA} \mathrm{CO}_{2}$ on geniohyoid muscle activity is in contrast to the excitatory effect of $\mathrm{CO}_{2}$ on UA muscle [3] and motor nerve activity [4] observed in adult cats. The authors have previously suggested that this excitatory effect on a UA dilator may stabilize the UA since UA narrowing or collapse might result in an increase in UA $\mathrm{CO}_{2}$ due to transfer of $\mathrm{CO}_{2}$ from the lungs. This in turn might restore UA patency through reflex contraction of UA dilators [3]. The presence of an opposite response of UA muscle activity to $\mathrm{UA} \mathrm{CO}_{2}$ in young guinea-pigs may increase the vulnerability of the UA to collapse in the young. However, it should be pointed out that the reflex effects of $\mathrm{UA} \mathrm{CO}_{2}$ in adult guinea-pigs are unknown.

The significance of the increase in ventilation is unclear. If such a reflex contributes to the overall ventilatory response to hypercapnia, then the pattern of the response may be protective against UA collapse since respiratory frequency rather than tidal volume is increased. In this way, ventilation is increased without increasing the collapsing pressure in the UA necessitated by an increase in tidal volume.

The absence of geniohyoid activity with intact vagi may suggest that this muscle and its response to $\mathrm{UA} \mathrm{CO}_{2}$ is unimportant in the regulation of UA resistance and patency in intact animals. However, anaesthesia preferentially depresses UA muscle activity compared to the diaphragm [21]. Furthermore, $\mathrm{UA} \mathrm{CO}_{2}$ reduced geniohyoid activity following vagotomy, and such a response would be expected to occur during UA occlusion and apnoea, when volume-related vagal afferent activity would be greatly reduced. 
All of these effects of UA $\mathrm{CO}_{2}$ on ventilation, UA resistance and UA muscle activity were abolished by anaesthesia of the UA, suggesting that they are due to reflex effects from $\mathrm{CO}_{2}$-sensitive receptors in the UA. A variety of $\mathrm{UA} \mathrm{CO}_{2}$-sensitive receptors have been identified in adult cats [1] and dogs [2], and $\mathrm{UA} \mathrm{CO}_{2}$ has been shown to exert reflex effects in adult rats [5] and cats [3, 4]. However, there is evidence that UA receptor function is immature in young animals [22] and it is not known whether $\mathrm{UA} \mathrm{CO}_{2}$-sensitive receptors are present in young animals. The present results are indirect evidence that such receptors are present in young guinea-pigs and that these receptors are functionally active.

It is not clear whether the effects on UA resistance and geniohyoid activity are specific reflex effects or whether they are secondary to the increase in ventilation. It is possible that the hypocapnia caused by the increase in ventilation may have reduced the drive to the UA dilator muscles including the geniohyoids, resulting in increased UA resistance.

To summarize, the present results show that upper airway carbon dioxide caused an increase in ventilation and upper airway resistance and a decrease in geniohyoid muscle activity in young guinea-pigs. All of these effects were abolished by topical anaesthesia of the upper airway, suggesting that they are reflex effects from carbon dioxidesensitive upper airway receptors.

Acknowledgements. The authors wish to thank T. Dowling and J. Slattery for technical assistance.

\section{References}

1. Bradford A, Nolan P, O'Regan RG, McKeogh D. Carbon dioxide-sensitive superior laryngeal nerve afferents in the anaesthetized cat. Exp Physiol 1993; 78: 787-798.

2. Anderson JW, Sant'Ambrogio FB, Orani GP, Sant' Ambrogio G, Mathew OP. Carbon dioxide-responsive laryngeal receptors in the dog. Respir Physiol 1990; 82: 217-226.

3. Nolan P, Bradford A, O'Regan RG, McKeogh D. The effects of changes in laryngeal airway $\mathrm{CO}_{2}$ concentration on genioglossus muscle activity in the anaesthetized cat. Exp Physiol 1990; 75: 271-274.

4. Bartlett D Jr, Knuth SL, Leiter JC. Alteration of ventilatory activity by intralaryngeal $\mathrm{CO}_{2}$ in the cat. $J$ Physiol 1992; 457: 177-185.

5. O'Halloran KD, Curran AK, Bradford A. Ventilatory and upper-airway resistance responses to upper-airway cooling and $\mathrm{CO}_{2}$ in anaesthetised rats. Eur J Physiol 1994; 429: $262-266$.
6. Curran AK, O'Halloran KD, Bradford A. Effects of superior laryngeal nerve section on ventilation in neonatal guinea-pigs. Respir Physiol 1995; 101: 23-29.

7. Al-Shway SF, Mortola JP. Respiratory effects of airflow through the upper airways in newborn kittens and puppies. J Appl Physiol 1982; 53: 805-814.

8. Boggs DF, Bartlett Jr. D. Chemical specificity of a laryngeal apneic reflex in puppies. J Appl Physiol 1982; 53: 455-462.

9. Fisher JT, Mathew OP, Sant'Ambrogio FB, Sant'Ambrogio G. Reflex effects and receptor responses to upper airway pressure and flow stimuli in developing puppies. $J$ Appl Physiol 1985; 58: 258-264.

10. Duara S, Rojas M, Claure N. Upper airway stability and respiratory muscle activity during inspiratory loading in fullterm neonates. J Appl Physiol 1994; 77: 37-42.

11. Dawes DS. Foetal and Neonatal Physiology. Chicago, IL, Year Book Medical Publishers, 1968; pp. 225-227.

12. Curran AK, O'Halloran KD, Bradford A. Developmental changes in the effect of airway $\mathrm{CO}_{2}$ on ventilation in anaesthetized guineapigs. $J$ Physiol (Lond) 1995; 489P: $163 \mathrm{P}$.

13. Zhang H-Q, Tai H-H, Lai J-L. Age-dependent mechanism in guinea pig bronchoconstriction induced by exsanguination. Respir Physiol 1995; 99: 361-369.

14. Curran AK, O'Halloran KD, Bradford A. Upper airway cooling reduces upper airway resistance in anaesthetized young guineapigs. Eur Respir J 1998; 11: 1257-1262.

15. O'Halloran KD, Curran AK, Bradford A. Effect of upper airway cooling and $\mathrm{CO}_{2}$ on diaphragm and geniohyoid muscle activity in the rat. Eur Respir J 1996; 9: 2323-2327.

16. Bartlett D Jr, Knuth SL, Gdovin MJ. Influence of laryngeal $\mathrm{CO}_{2}$ on respiratory activities of motor nerves to accessory muscles. Respir Physiol 1992; 90: 289-297.

17. Boushey HA, Richardson PS. The reflex effects of intralaryngeal carbon dioxide on the pattern of breathing. $J$ Physiol (Lond) 1973; 228: 181-191.

18. van Lunteren E, Dick TE. Breath-to-breath variability in hypoglossal motor unit firing. Respir Physiol 1992; 89: 37-46.

19. Brouillette RT, Thach BT. Control of genioglossus muscle inspiratory activity. J Appl Physiol 1980; 49: 801-808.

20. O'Halloran KD, Curran AK, Bradford A. Effect of almitrine on ventilation and on diaphragm and geniohyoid muscle activity in the rat. Clin Sci 1996; 91: 337-345.

21. Brouillette RT, Thach BT. A neuromuscular mechanism maintaining extrathoracic airway patency. J Appl Physiol 1979; 46: 772-779.

22. Miller AJ, Dunmire CR. Characterization of the postnatal development of superior laryngeal nerve fibres affecting swallowing and respiration. Exp Neurol 1974; 42: 369387. 